\title{
Imaging doses from the Elekta Synergy X-ray cone beam CT system
}

\author{
A. Amer, PhD, T. Marchant, PhD, J. Sykes ${ }^{*}$, MSc, J Czajka M.Sc, C. Moore, PhD
}

North Western Medical Physics, Christie Hospital NHS Trust, Manchester, M20 4BX, UK.

${ }^{*}$ Cookridge Hospital, Hospital Lane, Leeds, LS16 6QB.

This is the author's version of the work. It is posted here by permission of the BIR for personal use, not for distribution. The definitive version of was published in BJR (Volume 80, June 2007, DOI: $\underline{10.1259 / b j r / 80446730}$ )

\begin{abstract}
The Elekta Synergy is a radiotherapy treatment machine with integrated kilo-voltage $(\mathrm{kV}) \mathrm{X}$-ray imaging system capable of producing cone beam CT (CBCT) images of the patient in the treatment position. The aim of this study is to assess the additional imaging dose. Cone beam computed tomography dose index (CBDI) is introduced and measured inside standard CTDI phantoms for several sites (head: $100 \mathrm{kV}-38 \mathrm{mAs}$, lung: $120 \mathrm{kV}-152 \mathrm{mAs}$ and pelvis: $130 \mathrm{kV}-$ $456 \mathrm{mAs})$. The measured weighted doses were compared to TLD measurements at various locations in a Rando phantom and at patients' surfaces. The measured CBDIs in-air at isocentre were 9.2, 7.3 and $5.3 \mathrm{mGy} / 100 \mathrm{mAs}$ for 130,120 and $100 \mathrm{kV}$ respectively. The body phantom weighted CBDI were 5.5 and $3.8 \mathrm{mGy} / 100 \mathrm{mAs}$ for 130 and $120 \mathrm{kV}$. The head phantom weighted CBDI was $4.3 \mathrm{mGy} / 100 \mathrm{mAs}$ for $100 \mathrm{kV}$. The weighted doses for the Christie hospital CBCT imaging techniques were 1.6, 6 and 22mGy for the head, lung and pelvis. The measured CBDIs were used to estimate the total effective dose for the Synergy system using the ImPACT CT Patient Dosimetry Calculator. Measured CBCT doses using the Christie hospital protocols are low for head and lung scans whether compared with electronic portal imaging (EPI), commonly used for treatment verification, or single and multiple slice CT. For the pelvis, doses are similar to EPI but higher than CT. Repeated use of CBCT for treatment verification is likely and hence the total patient dose needs to be carefully considered. It is important to consider further development of low dose CBCT techniques to keep additional doses as low as reasonably practicable.
\end{abstract}




\section{Introduction}

The Elekta Synergy ${ }^{\mathrm{TM}}$ (Elekta Oncology Systems Ltd., Crawley, UK) is one of a new breed of radiotherapy linear accelerators specifically designed for image guided radiotherapy (IGRT). It has a kilo-voltage $\mathrm{X}$-ray source and opposing amorphous silicon flat panel imager, both mounted at $90^{\circ}$ to the treatment head for the acquisition of $\mathrm{kV}$ X-ray projection images for radiography and fluoroscopy, figure 1 . The different interaction mechanisms of kilo-voltage $(\mathrm{kV})$ photons with tissues and image transducers offer improved imaging compared to mega-voltage (MV) photons. This raises the prospect of enhanced localisation of target volumes and adjacent organs at risk, in the treatment room, compared to MV electronic portal imaging (EPI). Most importantly, the image sequence from rotation fluoroscopy can be used in filtered backprojection to reconstruct an X-ray volumetric image [1] . This form of cone beam computed tomography (CBCT) propels IGRT forward into a genuinely three dimensional (3D) technique and consequently has the potential for improving treatment setup and delivery [2-5].

In common with conventional trans-axial CT, the image quality for CBCT can be improved by increasing the number of $\mathrm{X}$-ray projections acquired and the mAs used. However, the IRMER legislation [6] embodies the principle of patient doses being 'as low as reasonably practical' (ALARP). In practice this translates to justifying the risks to the patient in the context of the likely benefits from imaging protocols that should be fit for purpose, but no more than this. Precedent sets the starting levels for doses that might be acceptable in CBCT. CT image sequences for radiotherapy planning (RTP-CT) and an MV verification image involve absorbed doses to various critical structures of 1-40mGy and 10-20mGy respectively [7]. However, with CBCT in IGRT there is the potential to image a patient at every fraction in order to support setup and the visualisation of the target/organs at risk (OAR) [3,8]. Guided solely by existing benchmarks the total additional dose resulting from serial use of CBCT could approach cautionary, deterministic levels. Since dose is added over a field of view wider than the target zone this has already influenced the debate on induced secondary cancers [7].

The Christie Hospital was one of the early sites selected to test the Synergy IGRT system which has been in clinical use since 2003. The aim of this paper is to report the likely patient doses arising from CBCT imaging protocols that have proven to be effective in clinical practice.

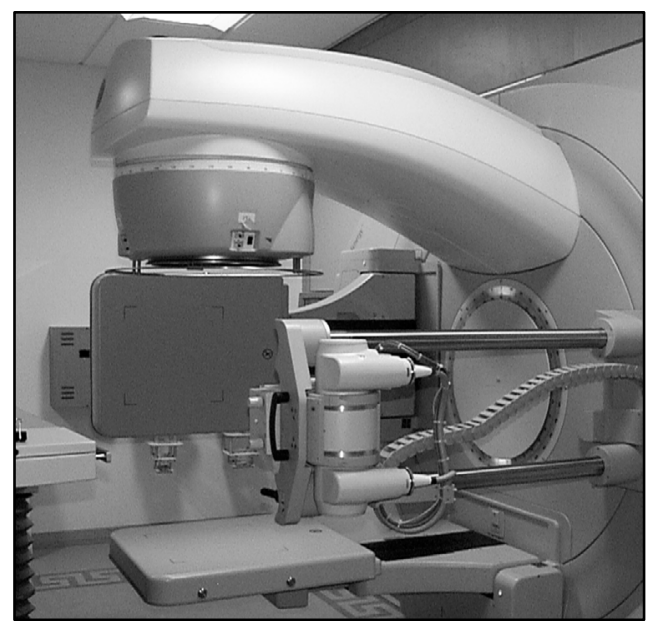

Figure 1: The Synergy system with retractable $\mathrm{kV} \mathrm{X}$-ray tube (right) and opposing $\mathrm{kV}$ imaging panel. 


\section{Theory}

European Guidelines on quality criteria for conventional CT imaging have been published by the European Commission [9]. The guidelines describe a reference dose level termed the weighted computed tomography dose index $\left(\mathrm{CTDI}_{\mathrm{w}}\right)$. This is a key component of the European dose reference levels (EDRL) for different anatomical sites. CTDI is simply the integral of the dose profile of a single slice along a line running parallel to the CT scanning axis, scaled by the nominal trans-axial slice thickness.

$$
C T D I=\frac{1}{L} \int_{-Z / 2}^{Z / 2} D(z) d z \quad[\mathrm{mGy}]
$$

where $\mathrm{D}(\mathrm{z})$ is the dose profile and $\mathrm{Z}$ is the integration range that covers most of the penumbra. The International Electrotechnical Commission (IEC) recommends $Z=10 \mathrm{~cm}$. The Dose profile integral (DPI) is usually measured using a $10 \mathrm{~cm}$ long ionization chamber in CTDI. L represents the nominal slice thickness (or the total thickness of more than one slice for multi-slice CT). For CBCT, L should represent the nominal length of the FOV in the axial direction and $\mathrm{Z}$ should be long enough to cover the penumbra region. This can be up to $40 \mathrm{~cm}$ for the Synergy system. Clearly, given the scale and that CBCT is not a sequential, slice based technique, CTDI is impractical for measuring dose in CBCT. Instead we suggest the continued use of the standard $10 \mathrm{~cm}$ chamber for CBCT dose measurement, whilst acknowledging that this method will not account for the dose in the penumbral region. Nevertheless, it will provide a reasonable measure of the dose in the central $10 \mathrm{~cm}$ region of the FOV. To distinguish this dose from CTDI, we will refer to it as cone-beam dose index (CBDI). In this case, L in equation (1) represents the chamber length of $10 \mathrm{~cm}$.

Similar to $\mathrm{CTDI}_{\mathrm{w}}, \mathrm{CBDI}_{\mathrm{w}}$ reflects the variation of dose deposition at depth by differentially weighting peripheral (p) and central (c) doses measured in standard CTDI phantoms.

$$
C B D I_{w}=\frac{1}{3} C B D I_{c}+\frac{2}{3} C B D I_{p} \quad[\mathrm{mGy}]
$$

The $\mathrm{CBDI}_{\mathrm{w}}$ is normalized to $100 \mathrm{mAs}$ by dividing the $\mathrm{CBDI}_{\mathrm{w}}$ by the exposure $\mathrm{E}$ (mAs) used to measure CBDI

$$
{ }_{n} C B D I_{w}=\frac{C B D I_{w} \times 100}{E} \quad[\mathrm{mGy} / 100 \mathrm{mAs}]
$$

\section{Materials and Methods}

\subsection{Synergy $k V X$-ray IGRT system}

The Elekta Synergy system, release 3.1, used in this study has a Dunlee X-ray tube (DU 304, Dunlee, USA) with a focal spot size of $1.2 \mathrm{~mm}^{2}$, located $100 \mathrm{~cm}$ from the centre of CBCT rotation. The tube has $1.5 \mathrm{~mm}$ Al equivalent inherent filtration and additional compound filtration of $1.5 \mathrm{~mm} \mathrm{Al}$ and $0.127 \mathrm{~mm} \mathrm{Cu}$. Tube potentials range from $40-130 \mathrm{kVp}$. Exposures are pulsed and range from 0.1-3.2mAs per X-ray projection. Two pairs of symmetric collimators (standard diaphragms) can be adjusted manually to shape the beam. The imaging transducer is of the 
indirect kind with a $\mathrm{Gd}_{2} \mathrm{O}_{2} \mathrm{~S}$ :Tb amorphous silicon (AmSi) flat panel (RID 1604, Perkin-Elmer Optoelectronics, Wiesbaden, Germany). It has an active area of $41 \times 41 \mathrm{~cm}^{2}$ addressed as an array of $1024 \times 1024$ pixels, each pixel having $400 \mu \mathrm{m}$ pitch. The panel is located $53.6 \mathrm{~cm}$ from the axis of rotation and images are captured at a fixed frame rate of $2.7 \mathrm{~Hz}$. Production Synergies are currently being installed with a CsI panel operating at higher frame rates. The latter will be the subject of a future, comparative investigation.

\subsection{Dose measurements}

At the outset it was regarded as important to attempt to identify the characteristic of skin and central doses that would assist practical prediction of patient related CBDI and the subsequent selection of the most efficient technique settings for clinical deployment. For the initial investigations, technique settings were: $100 \mathrm{kV}, 94 \mathrm{mAs}(25 \mathrm{~mA}, 10 \mathrm{~ms} /$ projection, nominal 380 projections) for the head; $130 \mathrm{kV}, 199 \mathrm{mAs}$ (40mA, 13ms/projection, nominal 380 projections) for the lung; and $130 \mathrm{kV}, 456 \mathrm{mAs}(40 \mathrm{~mA}$ and $30 \mathrm{~ms} /$ projection, nominal 380 projections) for the pelvis. Clinical investigations subsequently used the same settings for the pelvis but different settings $(100 \mathrm{kV}, 38 \mathrm{mAs})$ for the head and $(120 \mathrm{kV}$ and $152 \mathrm{mAs})$ for the lung. With IRMER in mind, these settings were found to be sufficient for IGRT at these sites. Measurements were taken to assess patient dose from the Synergy CBCT system using a Rando anthropomorphic phantom, standard CTDI phantom and patients. A $26 \mathrm{~cm}$-diameter and $26 \mathrm{~cm}-\mathrm{long}$ FOV in the axial direction was used for all dose measurements unless stated otherwise.

\subsubsection{Rando Phantom}

Lithium fluoride thermo-luminescent dosemeters (Harshaw TLD-100 LiF) were placed on the surface and at various depths inside a Rando phantom. Surface and internal doses to the head, lung and pelvis were investigated. The TLDs were read with a Harshaw 4500 reader (Harshaw Thermo Electron, Solon,USA). The TLDs were calibrated using a diagnostic X-ray set with 3mm Al filtration. The X-ray beam air kerma was measured with a 6cc chamber (Radcal model 90X66) and a Radcal 9010 radiation monitor. The instrument calibration is traceable to National Standards. The overall uncertainty in the TLD reading is $\pm 10 \%$ at $10 \mathrm{mGy}$ at the $95 \%$ confidence limit. Figure 2 shows a picture of the Rando phantom and the positions at which doses were measured for CBCT scanning. Each scan consisted of 380 projections taken during a $360^{\circ}$ gantry rotation. 

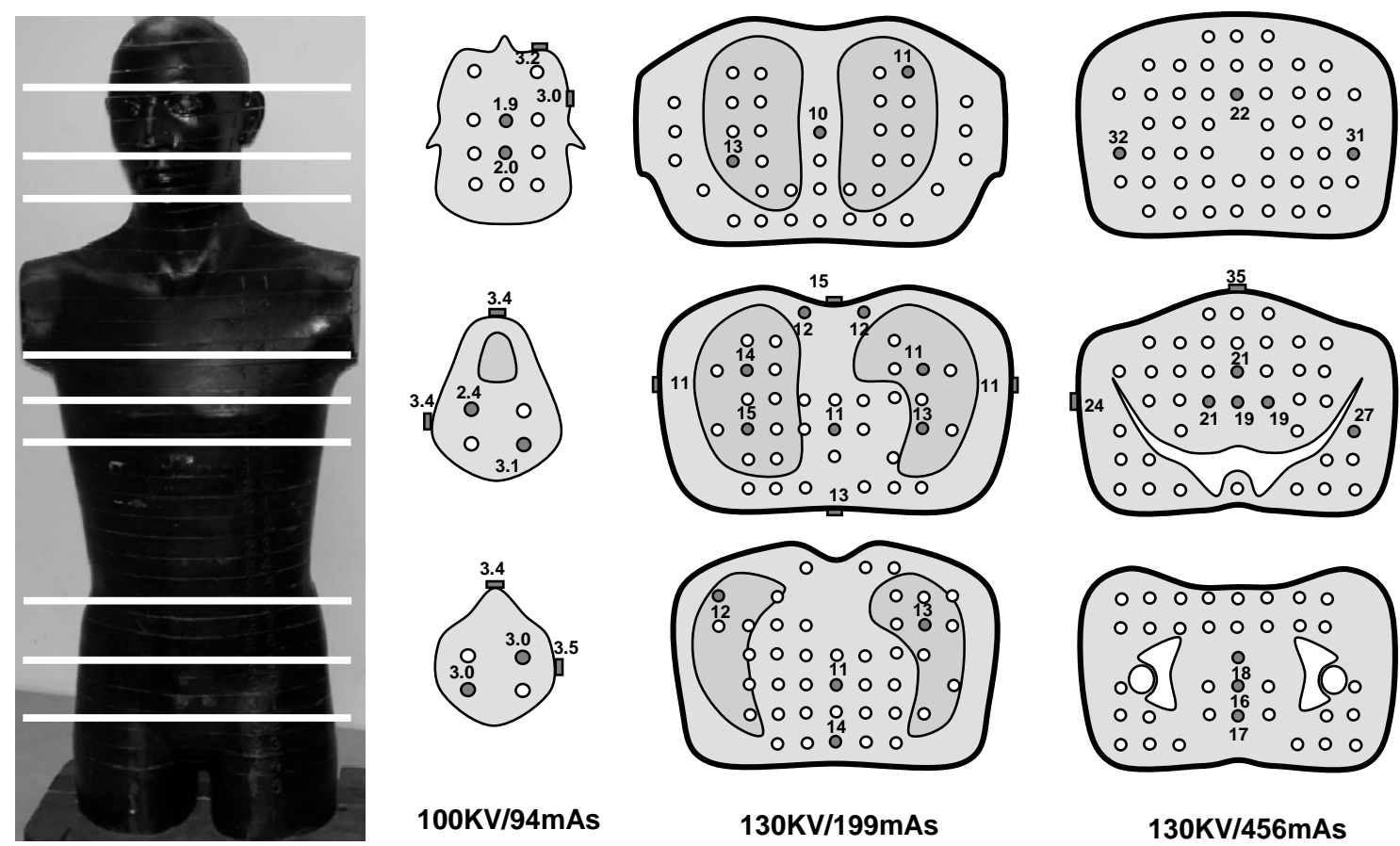

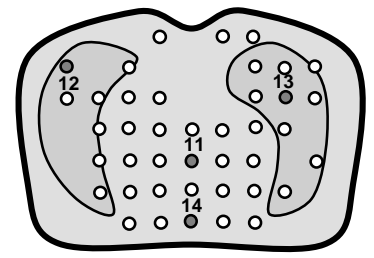

$130 \mathrm{KV} / 199 \mathrm{mAs}$

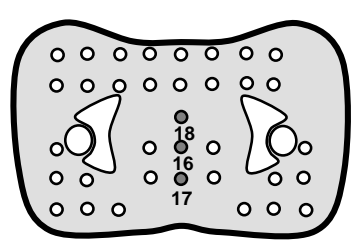

$130 \mathrm{KV} / 456 \mathrm{mAs}$

Figure 2: TLD measured dose [mGy] in the Rando phantom. White lines indicate the slices where doses were measured. The locations within each slice are indicated as black points, with the dose measurement itself adjacent. The $\mathrm{kV}$ and total $\mathrm{mAs}$ for each scan are indicated.

\subsubsection{CTDI phantom}

CBDIs were measured for a range of $\mathrm{kV}, \mathrm{mA}$ and ms settings in air and then inside standard, $14 \mathrm{~cm}$ long, 16 and $32 \mathrm{~cm}$ diameter head and body CTDI phantoms. In contrast to conventional slice-based CT scanners, which irradiate only short lengths of the patient at any given instant, the use of the standard $14 \mathrm{~cm}$ long CTDI phantoms does not cover the wide field CBCT and underestimates the CBDI due to missing scatter [10]. Hence, the effective length of the CTDI phantom was increased by adding $15 \mathrm{~cm}$ of Perspex (Plexiglass) to both ends of the body phantom and one end of the head phantom. A dose meter (Radcal 9010, Radcal Corp, Monrovia, CA, USA) with a $10 \mathrm{~cm}$ long, 3cc ion chamber was used. For the CBDI measured in air $\left(\mathrm{CBDI}_{\text {air }}\right)$, the ion chamber was placed at the centre of the CBCT rotation plane using the treatment machine alignment lasers, and with the chamber axis parallel to the axis of rotation. In the case of CBDI measured in a phantom, the centre of the phantom was positioned at the centre of rotation, again with axis parallel to that for rotation. Doses were then measured at the centre and periphery of the phantom for the standard $26 \mathrm{~cm}$ long FOV and also for collimated fields of view in the axial direction of 21 and $16 \mathrm{~cm}$.

A $0.125 \mathrm{~cm}^{3}$ ionisation chamber (PTW, Freiburg, Germany) was used to measure the central and peripheral dose profiles across the CTDI body phantom. This enables investigation of the shape of the dose profile, calculation of $\mathrm{CTDI}_{\mathrm{w}}$ and comparison with $\mathrm{CBDI}_{\mathrm{w}}$. The adequacy of using a $10 \mathrm{~cm}$ long ionisation chamber in CBCT dose measurements was assessed. 


\subsubsection{Patient Surface}

Point doses from CBCT scanning at patients' surfaces were measured using TLDs. Because of the $\mathrm{kV}$ energy of the CBCT X-ray beam, the dose at the surface represents the maximum dose that the patient receives. TLD measurements were taken for a total of 9 patients treated for head and neck, lung, bladder, prostate or cervix cancer with three to five TLDs per patient. These patients were from a pilot study looking at the use of cone beam 3-dimensional imaging in radiotherapy. The study had Local Research and Ethics Committee approval (South Manchester Local Research Ethics Committee approval number 02/SM/375). Informed written consent was obtained from all patients.

\subsection{ImPACT Dose Estimation}

The ImPACT CT Patient Dosimetry Calculator (ImPACT, London, UK) uses Monte Carlo generated dose data (NRPB SR-250 [11]) for a geometrical human model, and CTDI ${ }_{\text {air }}$ values for a particular scanning system, to estimate the absorbed dose to different organs and to calculate the total effective dose. The software package (version 0.99v) [12-14] was used to calculate these doses from the Synergy system. The doses are approximate, since the ImPACT calculator and the NRPB datasets do not take into account the divergent nature of a cone beam and so the shape of the dose distribution at the ends of the field is not accurately modelled. The Synergy system was matched to the nearest equivalent CT scanner within ImPACT database. The ratios of the CTDI, at centre and periphery, to air measurements allow the calculation of an empirically derived 'ImPACT factor' according to the formula available in the ImPACT calculator. The scanner with the closest factor having a flat beam filter as listed in NRPB SR-250, was selected.

\section{Results}

\subsection{CBDI measurements}

Figure 3 shows a longitudinal relative dose profile through more than half of a $26 \mathrm{~cm}$ long imaging field, measured using a $0.125 \mathrm{cc}$ chamber at central and peripheral positions, in a $32 \mathrm{~cm}$ diameter CTDI phantom with $15 \mathrm{~cm}$ Perspex scatter material added to both ends. The reduction in the dose profile at the periphery (inside the FOV) and the start of the sharp decline of the profile at $11 \mathrm{~cm}$ from the FOV centre, rather than $13 \mathrm{~cm}$ the nominal width, are attributable to the reduced scatter near the edge of the FOV and the divergent beam in CBCT. If one considers a dose profile from a full rotation scan, the dose profile at the periphery is influenced far more by the narrower entrance beam than the wider, highly attenuated exit beam. This is why the dose profile at the periphery is smaller than the nominal width for the field of view. By definition, the weighted dose is influenced more by the peripheral dose and hence the shape of the weighted dose profile is closer to the peripheral dose profile. Assuming symmetry of the relative dose profile, the weighted dose $\left(\mathrm{CBDI}_{\mathrm{w}}\right)$ across the central $10 \mathrm{~cm}$ of the FOV (the length of a standard ionisation chamber usually used in CTDI measurements) is $9 \%$ higher than the weighted dose $\left(\mathrm{CTDI}_{\mathrm{w}}\right)$ calculated from the integration of the weighted dose profile across $40 \mathrm{~cm}$ and divided by $26 \mathrm{~cm}$ (the nominal length of the imaging field). It can be seen from the relatively flat weighteddose profile of figure 3 that the use of a $10 \mathrm{~cm}$ chamber gives a useful, conservative overestimate of the weighted dose across the whole field of view (FOV). 


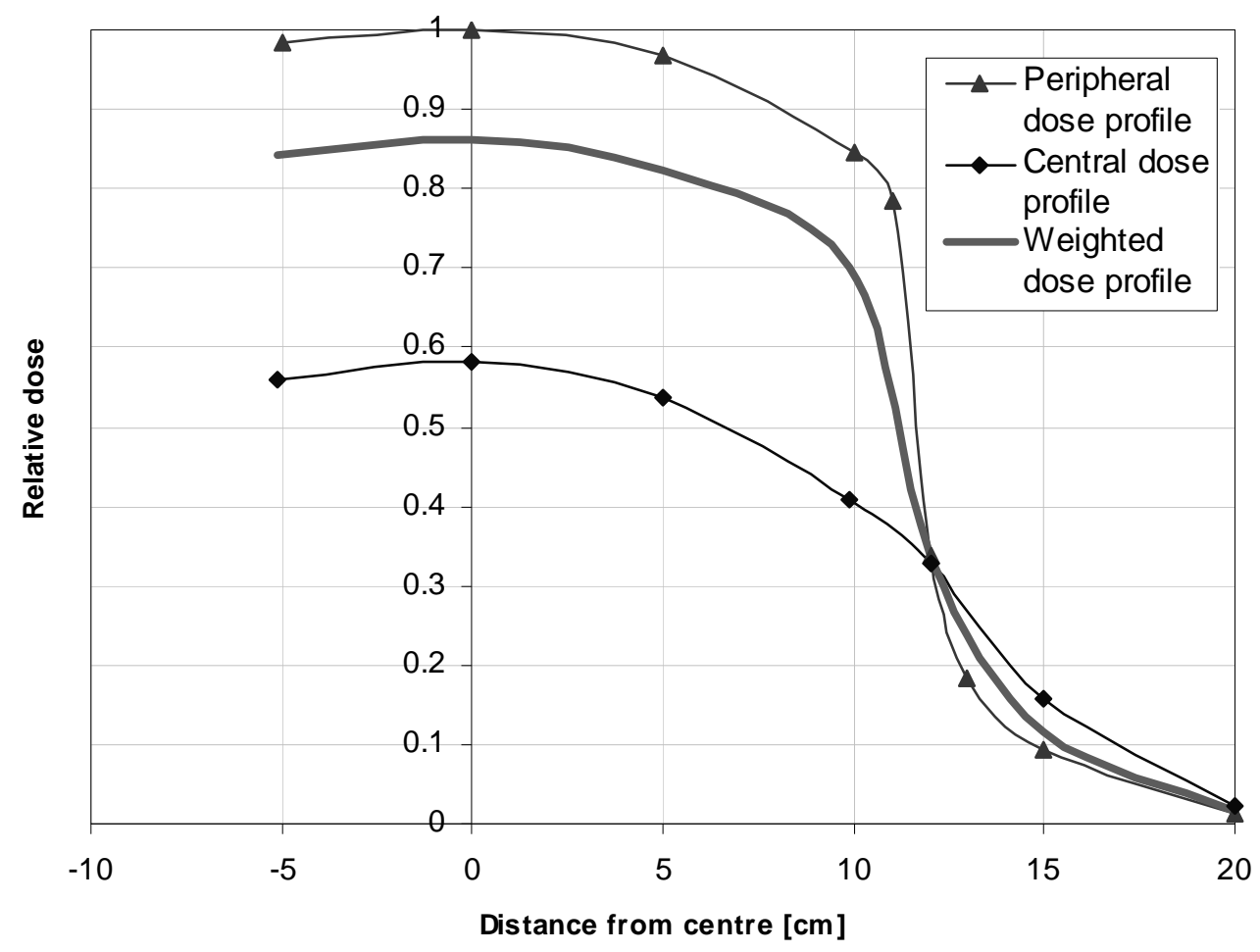

Figure 3: Longitudinal dose profiles across a $26 \mathrm{~cm}$ imaging field normalised to the maximum measured dose. The dose profiles were measured at central and peripheral positions in $32 \mathrm{~cm}$ diameter CTDI phantom with $15 \mathrm{~cm}$ additional scatter material to both ends of the phantom.

Table 1 shows the ${ }_{n} \mathrm{CBDI}_{\mathrm{w}}$ values measured for the beam settings adopted at the Christie Hospital. The $\mathrm{CBDI}_{\mathrm{w}}$ for $26 \mathrm{~cm}$ long FOV scans are 1.6, 6 and 25mGy for head, lung and pelvis respectively. The uncertainty in the CBDI measurements is $\pm 3 \%$ ( 1 standard deviation) estimated from repeated measurements. CBDI measurements obtained for the CTDI phantom without additional scatter material were lower, by $31 \%$ and $8 \%$ at the centre and periphery respectively, compared to those measured using additional scattering material on both sides. Beam collimation in the longitudinal direction reduces the contribution of scatter to CBDI. Compared to the $26 \mathrm{~cm}$ long FOV, 21 and $16 \mathrm{~cm}$ long FOVs resulted in 6 and $12 \%$ reduction in ${ }_{n} \mathrm{CBDI}_{\mathrm{w}}$ respectively

Table 1: Beam settings, nCBDIw values and weighted dose estimates for the clinical CBCT scanning protocols adopted at the Christie Hospital.

\begin{tabular}{cccccc}
\hline Site & \multicolumn{2}{c}{ Beam settings } & nCBDI_air & nCBDI_w & CBDI_w \\
& $\mathbf{k V}$ & $\mathbf{m A s}$ & $\mathbf{( m G y / 1 0 0 m A s )}$ & $\mathbf{( m G y / 1 0 0 m A s )}$ & $\mathbf{( m G y )}$ \\
\hline Head & 100 & 38 & 5.3 & 4.3 (head) & 1.6 \\
\hline Lung & 120 & 152 & 7.3 & 3.8 (body) & 6 \\
\hline Pelvis & 130 & 456 & 9.2 & 5.5 (body) & 25 \\
\hline
\end{tabular}




\subsection{Rando and Patient doses}

Figure 2 shows the TLD dosimetry results at different locations in the Rando phantom. The average doses from CBCT at the anterior and lateral surface of several cancer patients are provided in Table 2. The Rando and patients' measured point doses were normalised to the adopted imaging beam settings (Dose normalised $=$ Dose $\left._{\text {measured }} \times\left(\mathrm{mAs} / \mathrm{mAs}_{\text {adopted }}\right) \times\left(\mathrm{kV} / \mathrm{kV}_{\text {adopted }}\right)^{2}\right)$ for comparison, and are given in Table 3 . There is very good agreement between the external doses measured at the surface for Rando and patients. Hence, the internal Rando point doses represent a good estimate of the patient internal point doses.

Table 2: Summary of the TLD measured dose in mGy at the surface of patients imaged with CBCT.

\begin{tabular}{cccccc}
\hline \multirow{2}{*}{ Patient } & \multirow{2}{*}{ Site } & $\begin{array}{c}\text { TLDs / } \\
\text { patient }\end{array}$ & mAs & \multicolumn{2}{c}{ Dose at surface (mGy) } \\
Anterior & Lateral \\
\hline 1 & Prostate & 3 & 440 & 32 & 21 \\
2 & Prostate & 3 & 440 & 34 & 22 \\
3 & Bladder & 5 & 440 & 33 & 21 \\
4 & Prostate & 2 & 440 & 35 & 23 \\
5 & Cervix & 4 & 440 & 35 & 21 \\
6 & H\&N & 3 & 90 & 3.0 & 2.9 \\
7 & H\&N & 2 & 90 & 2.7 & 2.8 \\
8 & Lung & 2 & 200 & 13 & 7.6 \\
9 & Lung & 3 & 200 & 15 & 8.1 \\
\hline
\end{tabular}

Table 3: Comparison of the Patients, Rando and ImPACT doses in mGy at different locations normalised to the beam settings of the adopted imaging protocols.

\begin{tabular}{clccc}
\hline Site (beam settings) & Position & $\begin{array}{c}\text { Patient } \\
\text { mGy }\end{array}$ & $\begin{array}{c}\text { Rando } \\
\text { mGy }\end{array}$ & $\begin{array}{c}\text { ImPACT } \\
\text { mGy }\end{array}$ \\
\hline \multirow{2}{*}{ Head } & Surface (Ant) & 1.2 & 1.3 & - \\
$(100 \mathrm{kV} / 38 \mathrm{mAs})$ & Surface (Lat) & 1.2 & 1.2 & - \\
& Eye lens & - & 1.3 & 1.2 \\
\hline \multirow{2}{*}{ Lung } & Surface (Ant) & 11 & 10 & - \\
$(120 \mathrm{kV} / 152 \mathrm{mAs})$ & Surface (Lat) & 6.0 & 7.2 & - \\
& Heart & - & 7.2 & 7.1 \\
\hline \multirow{2}{*}{ Pelvis } & Surface (Ant) & 35 & 34 & - \\
$(130 \mathrm{kV} / 456 \mathrm{mAs})$ & Surface (Lat) & 22 & 23 & - \\
& Uterus & - & 21 & 23 \\
\hline
\end{tabular}




\subsection{ImPACT dose estimation}

Based on the CBDI measurements, Synergy was matched to the Siemens Somatom, DRH, CR conventional scanner. Using the adopted imaging beam settings, the ImPACT calculator estimated doses to be 1.2, 7.1 and 23mGy for eye lenses, heart and uterus. These are in good agreement with 1.3, 7.8 and 21mGy measured using TLDs in Rando after normalisation to the adopted technique settings. The total effective dose calculated using the ImPACT software were $0.1,1.6$ and $6 \mathrm{mSv}$ for the head, lung and pelvis scans respectively.

\section{Discussion}

The choice of the nominal FOV width for CBCT beam is not a clear cut because of the divergence of the conical beam. We feel that the width of the beam at the axis of rotation gives a balanced choice in terms of dose assessment and available useful image information. Figure 4 (a) shows the entrance, isocentre and exit beam widths arising from rotational scanning with a conical beam. There are always a full set of projections for points on the conical apices defining the scanned volume. Additionally, there are sufficient projections from most of the scanning directions for a good if not perfect reconstruction of adjacent points. When viewed non-axially, a $26 \mathrm{~cm}$ field of view clearly provides a substantial amount of useful information, well beyond the entrance width. This is demonstrated in figure 4 (b) which is a coronal $256 \times 256 \mathrm{~mm}$ CBCT image. On this image the edges of the divergent X-ray beam used for cone beam scanning are indicated. It is of course possible to exclude areas not reconstructed from a complete set of projection angles, but we feel this is short sighted, since it discards useful and available data! 
(a)

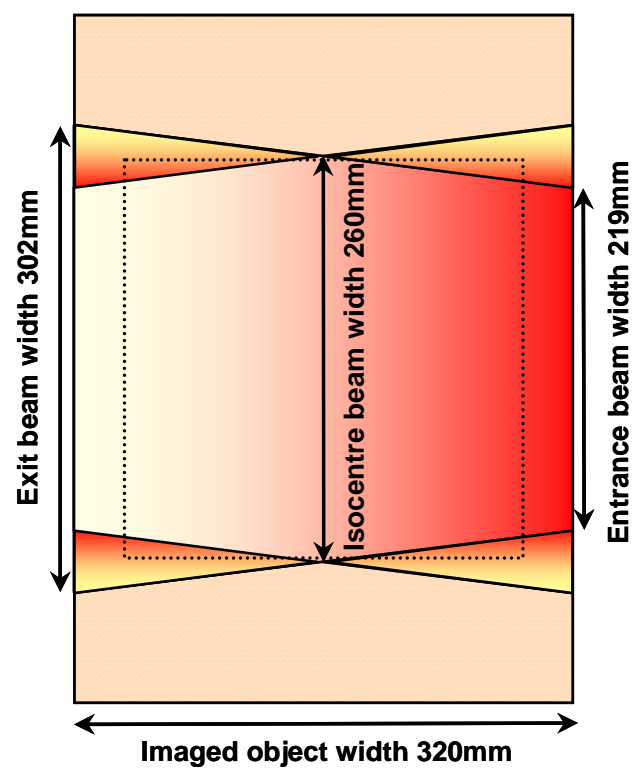

(b)

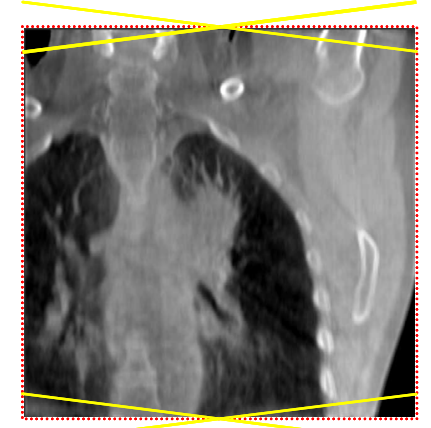

Figure 4: (a) A schematic diagram of the entrance, isocentre and exit beam width when imaging a $32 \mathrm{~cm}$ wide object. (b) A coronal $256 \times 256 \mathrm{~mm}$ image of a lung cancer patient showing useful information beyond the nominal entrance beam width.

In the case of CTDI dose measurements for diagnostic CBCT, Mori, et. al. [10] pointed out the need for a longer CTDI phantom and ionisation chamber. Our results from the Synergy system strongly reinforce the need for a longer CTDI phantom (or the addition of scattering material) in radiotherapy $\mathrm{CBCT}$, where the image volume is generated from a single rotation of a genuinely wide-angle cone beam. However, from the measured shape of the weighted dose profile across the CTDI phantom, figure 3 , the use of a $10 \mathrm{~cm}$ chamber appears to give a conservative overestimate measure of the weighted dose across the imaged volume.

Currently, patient position verification is commonly done by acquiring EPI using the megavoltage X-ray treatment beam. These images are acquired using the exit treatment beam but in many cases the limited field provides insufficient detail to register to the digitally reconstructed radiograph (DRR). Hence, EPIs with wider fields are often taken before treatment. At our institution, linear accelerators are calibrated to produce a point dose of 1cGy per monitor unit at the depth of maximum dose for a $10 \mathrm{~cm} \times 10 \mathrm{~cm}$ field and a $100 \mathrm{~cm}$ focal to surface distance. EPIs are usually acquired with 2-4 monitor units per image and often two orthogonal EPIs are needed to verify the patient position in the three cardinal directions. Approximating the average patient dose to half of the maximum, then the average dose from verification images is typically 20-40mGy per pair of images. The CBDI dose from CBCT is less than that from wide-field EPI, especially for head and lung scans. This advantage, in addition to the better image quality using $\mathrm{kV} \mathrm{X}$-rays and the 3D information it provides, suggest it may become an important modality for verification in the future.

The European directive [15] and UK regulations [6], require the justification and optimisation of dose used in medical imaging. The justification for the extra dose from CBCT could be the 
reduction of uncertainty in the process of treatment delivery which promises higher rates of tumour control and/or lower rates of complication. Nevertheless, the CBCT image quality should always be optimised for a given purpose. For example, verification of head and neck position can be achieved with a lower CBCT dose ( 1mGy), sufficient to show bony details to register with the planning scan [16], than that required for the visualisation of tumours or soft tissue structures in the pelvis ( $\sim 25 \mathrm{mGy})$. Other methods should be sought to keep the dose as low as practically achievable.

More consideration is required when contemplating repeated or serial use of CBCT. For example, a typical CBCT imaging protocol for pelvis would result in patient surface dose of $30 \mathrm{mGy}$ per scan $[8,17]$. In the case of an online correction protocol or clinical studies that require imaging on a daily basis (40 fractions), the total surface dose approaches the deterministic level for transient skin erythema of 2Gy [18]. Caution should be exercised when imaging sites such as breast, particularly in younger women, where the skin dose from radiotherapy alone is already of clinical concern [19]. For head and neck, ocular opacities have a threshold of only 500mGy [18]

Figure 5 shows image quality for CBCT imaging protocols adopted at the Christie Hospital. The CBDI from these protocols are very low for the head and lung scans compared with the CTDI of two recent surveys conducted in the UK and Europe for single and multi-slice CT [20, 21]. The CBDI from pelvic scans is higher due to the need to see soft tissue contrast in what is after all a high scatter imaging modality. Table 4 gives a comparison of the CBDI with the CTDI of the two surveys.

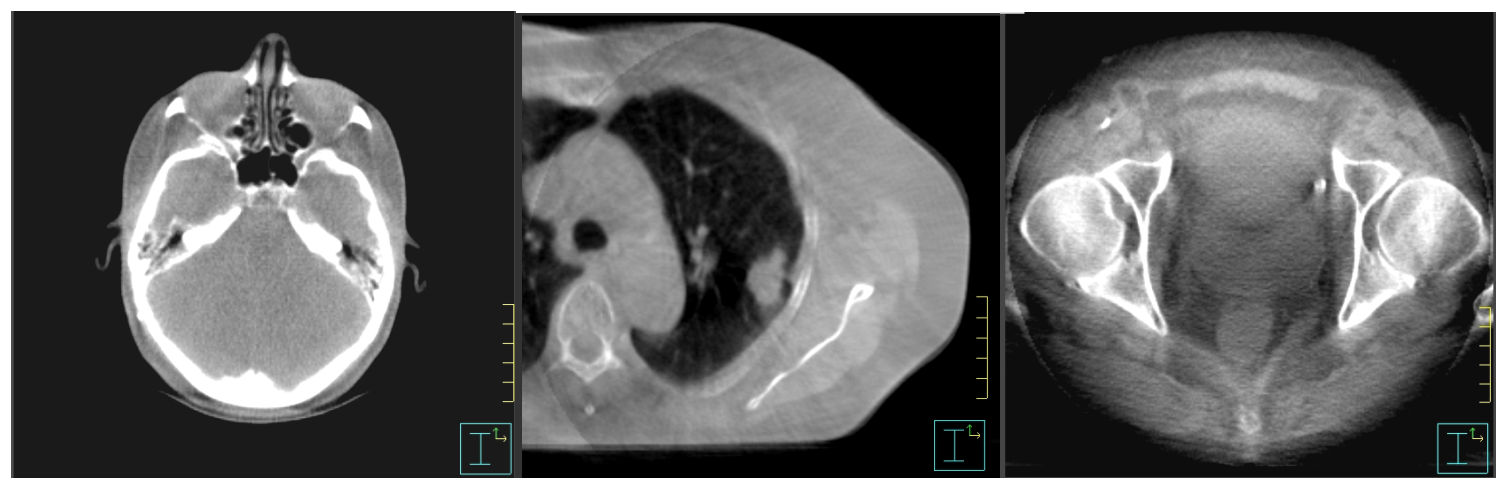

Figure 5: Representative axial slices through CBCT image volumes of head (left), lung (middle) and prostate (right) showing the image quality with the Christie Hospital imaging protocols. Effective slice widths are $3 \mathrm{~mm}$ for the head and $5 \mathrm{~mm}$ for lung and pelvis. 
Table 4: Comparison of the cone beam dose index (CBDI) in mGy with UK and European

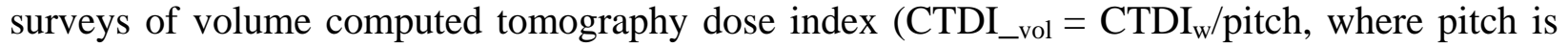
the ratio of the table feed and slice thickness).

\begin{tabular}{l|c|cc|c}
\hline \multirow{2}{*}{} & \multirow{2}{*}{$\begin{array}{c}\text { Europe } \\
\text { MSCT }\end{array}$} & \multicolumn{2}{|c|}{ UK } & Christie \\
& CTCT & MSCT & CBCT \\
CTDI_vol[mGy] & CTDI_vol[mGy] & CBDI [mGy] \\
\hline Lung & 77 & 59 & 80 & $\mathbf{1 . 6}$ \\
Pelvis & 12 & 10 & 13 & $\mathbf{6}$ \\
\hline
\end{tabular}

Abreviations: Single slice CT (SSCT), multi slice CT (MSCT), cone beam CT (CBCT).

${ }^{\mathrm{a}}$ Dose from a collimated FOV in the longitudinal direction $(16 \mathrm{~cm})$ used clinically.

\section{Conclusion}

Measurements of CBCT dose made in Rando, CTDI phantom and patients were generally consistent with each other. For image acquisition settings adopted at the Christie Hospital, CBCT doses from the Synergy image guided radiotherapy system are low for sites such as head and lung, by factors $>10$ and 2 respectively, compared to conventional CT. For sites where soft tissue details are needed, such as the pelvis, doses are $~ 50 \%$ higher than those seen in conventional CT and similar to the dose from orthogonal EPIs minimally required for 3D setup verification. 


\section{References}

1. Jaffray DA, Siewerdsen JH, Wong JW, Martinez AA. Flat-panel cone-beam computed tomography for image-guided radiation therapy. International Journal of Radiation Oncology*Biology*Physics 2002;53(5):1337-1349.

2. Ghilezan M, Yan D, Liang J, Jaffray DA, Wong JW, Martinez AA. Online image-guided intensity-modulated radiotherapy for prostate cancer: How much improvement can we expect? A theoretical assessment of clinical benefits and potential dose escalation by improving precision and accuracy of radiation delivery. International Journal of Radiation Oncology*Biology*Physics 2004;60(5):1602.

3. Oldham M, Letourneau D, Watt L, Hugo G, Yan D, Lockman D, et al. Cone-beam-CT guided radiation therapy: A model for on-line application. Radiotherapy and Oncology 2005;75(3):271.E1.

4. Jaffray DA. Emergent Technologies for 3-Dimensional Image-Guided Radiation Delivery. Seminars in Radiation Oncology 2005;15(3):208.

5. Yan D, Lockman D, Martinez AA, Wong JW, Brabbins D, Vicini F, et al. Computed Tomography Guided Management of Interfractional Patient Variation. Seminars in Radiation Oncology 2005;15(3):168.

6. HMSO. Ionising Radiation (Medical Exposure) Regulations. In: SI 2000/1059. UK: HMSO; 2000.

7. Aird EG. Second cancer risk, concomitant exposures and IRMER(2000). British Journal of Radiology 2004;77(924):983-5.

8. Letourneau D, Wong JW, Oldham M, Gulam M, Watt L, Jaffray DA, et al. Cone-beam-CT guided radiation therapy: technical implementation. Radiotherapy and Oncology 2005;75(3):279-286.

9. EU. European Guidelines on Quality Criteria for Computed Tomography. Brussels: EU; 1999. Report No.: EUR 16262.

10. Mori S, Endo M, Nishizawa K, Tsunoo T, Aoyama T, Fujiwara H, et al. Enlarged longitudinal dose profiles in cone-beam CT and the need for modified dosimetry. Medical Physics 2005;32(4):1061-9.

11. Jones DG, Shrimpton PC. Normalised organ doses for X-ray computed tomography calculated using Monte Carlo techniques. Oxford: NRPB. Report No.: SR250.

12. ImPACT - CT patient dosimetry calculator. www.Impactscan.org. St George's Hospital, London, UK; 2005.

13. Shrimpton PC, Jones DG, Hillier MC, Wall BF, Le Heron JC, Faulkner K. Survey of CT practice in the UK. Part 2: Dosimetric aspects.: NRPB; 1991. Report No.: R249.

14. Jones DG, Shrimpton PC. Survey of CT practice in the UK. Part 3: Normalised organ doses calculated using Monte Carlo techniques.: NRPB; 1991. Report No.: R250.

15. EU. Council Directive 97/43/Euratom. In: Health Protection of Individuals Against the Dangers of Ionizing Radiation in relation to Medical Exposure. EU; 1997.

16. Sykes JR, Amer A, Czajka J, Moore CJ. A feasibility study for image guided radiotherapy using low dose, high speed, cone beam X-ray volumetric imaging. Radiotherapy and Oncology 2005; 77: 45-52.

17. Smitsmans MHP, de Bois J, Sonke J-J, Betgen A, Zijp LJ, Jaffray DA, et al. Automatic prostate localization on cone-beam CT scans for high precision image-guided radiotherapy. International Journal of Radiation Oncology*Biology*Physics 2005;63(4):975.

18. Valentin J. Radiation and your patient: A guide for medical practitioners: ICRP Supporting Guidance 2. Annals of the ICRP 2001;31(4):1.

19. Woo TCS, Pignol J-P, Rakovitch E, Vu T, Hicks D, O'Brien P, et al. Body radiation exposure in breast cancer radiotherapy: impact of breast IMRT and virtual wedge compensation 
techniques. International Journal of Radiation Oncology*Biology*Physics; In Press, Corrected Proof.

20. Shrimpton PC, Hillier MC, Lewis MA, Dunn M. Doses from Computed Tomography (CT) Examinations in the UK - 2003 Review. Chilton, UK.: NRPB-W67; 2005. Report No.: NRPB-W67.

21. MSCT. 2004 CT Quality Criteria. Results from a European Concerted Action on CT (FIGMCT-2000-20078). published at http://www.msct.info/CT_Quality_Criteria.htm; 2004. 